\section{Uso de plantas medicinais e fitoterápicos entre usuários de uma clínica universitária de fisioterapia do noroeste do Rio Grande do Sul}

\section{Use of medicinal plants and phytotherapics among users of a physiotherapy clinic in the northwest of Rio Grande do Sul}

\author{
Laís Corrêa da Cunha, Viviane Cecília Kessler Nunes Deuschle, Regis Augusto Norbert \\ Deuschle
}

Como citar este artigo:

DA CUNHA, LAÍS C.; DEUSCHLE, VIVIANE C. K. N.; DEUSCHLE, REGIS

A. N.; Uso de plantas medicinais e fitoterápicos entre usuários de uma clínica universitária de fisioterapia do noroeste do Rio Grande do Sul. Revista Saúde (Sta. Maria). 2021; 47 (1).

\section{Autor correspondente: \\ Nome: Laís Corrêa da Cunha E-mail: lais-correag@hotmail.com Formação Profissional: Formada em Farmácia pela Universidade de Cruz Alta (UNICRUZ) que fica na cidade de Cruz Alta, RS, Brasil. \\ Filiação Institucional: Universidade de Cruz Alta \\ Endereço para correspondência: \\ Rua: Rodovia Municipal Jacob \\ Della Méa \\ Bairro: Distrito Parada Benito \\ Cidade: Cruz Alta \\ Estado: Rio Grande do Sul \\ CEP: 98020290}

Data de Submissão:

24/07/2020

Data de aceite:

10/05/2021

Conflito de Interesse: Não há conflito de interesse

\section{(cc) $\mathrm{BY}-\mathrm{NC}-\mathrm{ND}$}

\begin{abstract}
RESUMO
$\mathrm{O}$ uso de plantas medicinais para a prevenção e tratamento de doenças data de muitos anos, sendo a sua utilização perpetuada em maior ou menor grau através das gerações. A relativa facilidade de acesso e 0 baixo custo estão entre os fatores que impulsionam o uso pela população. Aliadas aos fitoterápicos, constituem bens sociais muito utilizados pela população brasileira. Entretanto, para que as ações de saúde que trabalhem o uso racional destes recursos possam ser direcionadas a uma população deve-se primeiro conhecer como eles são utilizados. 0 objetivo do estudo foi analisar o uso de plantas medicinais e medicamentos fitoterápicos pelos usuários de uma clínica universitária de fisioterapia do noroeste do Rio Grande do Sul. Foi realizado um estudo observacional descritivo transversal com a aplicação de questionário estruturado com perguntas abertas e fechadas, o qual foi aplicado a uma população de 25 indivíduos no mês de novembro de 2019. Observou-se que 100\% dos participantes utilizavam plantas medicinais, sendo a marcela e camomila as mais frequentes. Apenas $4 \%$ dos entrevistados informaram já ter utilizado medicamento fitoterápico. Entre os participantes, $96 \%$ informaram acreditar que as plantas medicinais não possuem efeitos adversos, $64 \%$ informaram que possuem a planta usada cultivada em sua própria residência e $92 \%$ aprenderam a utilizá-las através de ensinamentos de parentes. Foi analisada na literatura a possível existência de interações entre os medicamentos e as plantas medicinais utilizadas pelos participantes, encontrando-se interações com medicamentos antihipertensivos, antiinflamatórios e antidiabéticos. Para algumas plantas citadas pelos entrevistados não foi encontrado embasamento científico para as indicações para as quais eram utilizadas. Devido ao uso difundido das plantas medicinais na população, observa-se a necessidade de uma maior contribuição dos profissionais da saúde para orientar sobre 0 uso racional dos produtos naturais.
\end{abstract}

PALAVRAS-CHAVE: Plantas medicinais; Fitoterapia; Atenção Farmacêutica.

\section{ABSTRACT}

The use of medicinal plants for the prevention and treatment of diseases dates back many years, and their use has been perpetuated to a greater or lesser degree through the generations. Relative ease of access and low cost are among the factors that drive its use by the population. Allied to phytotherapics, they are social goods widely used by the Brazilian population. The aim of the study was to analyze the use of medicinal plants and herbal medicines by users of a university physiotherapy clinic in the northwest of Rio Grande do Sul. A cross-sectional observational study was conducted with the application of a structured questionnaire with open and closed questions, which was applied to a population of 25 individuals in November 2019. It was observed that $100 \%$ of the participants used medicinal plants, with marcela and chamomile being the most frequent. Only $4 \%$ of respondents reported having used herbal medicine. Among the participants, $96 \%$ reported believing that medicinal plants do not have adverse effects, $64 \%$ reported that they have the used plant grown in their own residence and $92 \%$ learned to use them through the teachings of relatives. The possible existence of interactions between drugs and medicinal plants used by the participants was analyzed in the literature, with interactions with antihypertensive, anti-inflammatory and anti-diabetic drugs. For some plants mentioned by the interviewees, no scientific basis was found for the indications for which they were used. Due to the widespread use of medicinal plants in the population, there is a need for a greater contribution from health professionals to advise on the rational use of natural products.

KEYWORDS: Medicinal plants; Phytotherapy; Pharmaceutical attention. 


\section{INTRODUÇÃO}

As plantas medicinais são definidas pela Organização Mundial da Saúde como "todo e qualquer vegetal que possui, em um ou mais órgãos, substâncias que podem ser utilizadas com fins terapêuticos ou que sejam precursores de fármacos semissintéticos"1. Medicamento fitoterápico é definido pela Anvisa como medicamento "obtido com emprego exclusivo de matérias-primas ativas vegetais cuja segurança e eficácia sejam baseadas em evidências clínicas e que sejam caracterizados pela constância de sua qualidade"2.

Os medicamentos fitoterápicos e as plantas medicinais se apresentam como recursos de prevenção e tratamento de doenças. Em relação às plantas medicinais, o baixo custo e a facilidade de acesso estão entre os fatores que impulsionam o uso pela população ${ }^{3}$. O uso popular suscita estudos na busca da definição das propriedades biológicas das plantas e seus derivados. A partir destes trabalhos, há uma crescente descoberta de compostos fitoquímicos com propriedades farmacológicas proeminentes, algumas vezes demonstrando a efetividade encontrada no uso empírico ${ }^{4}$.

A grande diversidade vegetal presente no país propiciou, desde seu passado até os dias de hoje, a busca e o uso de recursos naturais, contribuindo para que conhecimentos fossem acumulados e passados de geração em geração. Na sociedade atual, que sofre constantes pressões econômicas e é marcada pelo conhecimento científico e tecnológico, o uso das plantas medicinais como alternativa terapêutica é de relevância. Nesse sentido, é necessário que cada vez mais estudos de efetividade sejam realizados, contribuindo para enaltecer a cultura e diversidade das regiões e também propiciando o uso seguro de produtos naturais com fins terapêuticos devidamentes embasados ${ }^{5}$.

Quando utilizados de forma racional, as plantas medicinais e medicamentos fitoterápicos constituem recursos valiosos. Porém, quando utilizados dentro da perspectiva da prática da automedicação, podem resultar em complicações para a saúde ${ }^{6}$. A automedicação é bastante comum e está associada a vários fatores, tais como a facilidade de acesso a uma ampla gama de medicamentos e plantas medicinais. Produtos naturais também são procurados pela ideia de inocuidade, ou seja, que não irão ocasionar efeitos adversos, interações e intoxicações ${ }^{7}$. $O$ baixo custo, combinado com a dificuldade do acesso a consultas nos sistemas de saúde, são fatores adicionais que contribuem com essa prática pela população $0^{8,9,10}$.

Contudo, a automedicação pode ocasionar resultados indesejáveis ao paciente, tais como ineficácia, reações adversas, interações medicamentosas, intoxicações, alergias entre outras complicações ${ }^{11}$. A literatura demonstra que produtos naturais podem apresentar interações medicamentosas com vários fármacos. Cita-se o exemplo do Rosmarinus officinalis L., alecrim, que pode diminuir a ação de diuréticos, laxantes e hipotensores ${ }^{12}$. A espécie Pimpinella anisum L., erva-doce, possui interação com drogas hipnóticas, prolongando os seus efeitos ${ }^{12}$.

Desta forma, para que as ações de saúde que trabalhem o uso racional destes recursos possam ser direcionadas a uma população, evitando complicações indesejáveis, deve-se primeiro conhecer como eles são utilizados. Portanto, 
o objetivo deste trabalho é analisar o uso de plantas medicinais e medicamentos fitoterápicos pelos usuários de uma clínica universitária de fisioterapia do noroeste do Rio Grande do Sul, listar as plantas medicinais e fitoterápicos utilizados pelos pacientes e as indicações atribuídas por eles, assim como suas formas de uso. Também objetiva-se comparar as indicações atribuídas pelos usuários às plantas medicinais/fitoterápicos com a literatura científica e analisar a possibilidade de interações entre os fitoterápicos/plantas medicinais com os fármacos em uso.

\section{MÉTODO}

Estudo observacional descritivo onde foi estudado o hábito do uso de plantas medicinais e fitoterápicos entre pacientes da clínica de fisioterapia de uma universidade comunitária em Cruz Alta, no Noroeste do Rio Grande do Sul, mediante aplicação de um questionário estruturado com perguntas abertas e fechadas. 0 estudo foi aprovado pelo Comitê de Ética em Pesquisa da Universidade de Cruz Alta - Unicruz sob o parecer consubstanciado 3.671.086 e CAAE 20181121162559 .

A aplicação do questionário foi realizada no período de novembro de 2019, em uma população de 25 pacientes. Foram incluídos todos os pacientes cadastrados na clínica de fisioterapia, com faixa etária de 18 a 90 anos, de ambos os sexos. Foram excluídas do estudo as pessoas submetidas a tratamento na clínica em período inferior a um mês. Também foram excluídos pacientes que não realizaram uso de medicamentos nos últimos 15 dias.

O questionário foi dividido em quatro seções: a primeira consistiu na caracterização socioeconômica; a segunda, no uso pregresso de medicamentos (fitoterápicos inclusos); a terceira, no uso atual; e a quarta, especificamente sobre plantas medicinais.

Os dados foram analisados em planilha eletrônica aplicando-se estatística descritiva, com os resultados apresentados em porcentagem. Relatos em perguntas abertas foram registrados em editor de texto.

\section{RESULTADOS E DISCUSSÃO}

Foram entrevistados 25 pacientes da clínica. Dentre os participantes, $56 \%$ eram do sexo feminino e $44 \%$ eram do sexo masculino. Dentre os participantes, $96 \%$ eram da cidade de Cruz Alta e 4\% da cidade de Panambi. A idade dos entrevistados variou de 22 a 80 anos, com a maioria na faixa etária entre 41 a 60 anos (44\%), seguido de $32 \%$ na faixa de 61 a 83 e 24\% na faixa de 22 a 40 anos. Quanto ao grau de instrução dos participantes, $40 \%$ concluíram o ensino médio e $20 \%$ possuíam ensino superior completo. $28 \%$ relataram ser aposentados. Em relação a renda familiar, $70 \%$ relataram possuir renda entre 1 a 3 salários mínimos. Ao pesquisar o meio de comunicação mais utilizado pelos participantes, $68 \%$ informaram utilizar a internet via celular como principal meio. Desta forma, tem-se uma população predominantemente adulta com aproximadamente um terço na terceira idade, com renda relativamente baixa e um razoável nível de instrução. 
Considera-se que estes fatores podem contribuir com a busca por plantas medicinais como recursos terapêuticos pelos motivos de fácil acesso e baixo custo. Considera-se ainda que o grupo estudado já é portador de alguma patologia, uma vez que são pacientes de uma clínica de fisioterapia, sob terapia músculo-esquelética no momento da realização do estudo.

Ao questionar sobre o conhecimento e utilização de plantas medicinais, $100 \%$ dos entrevistados informaram utilizar plantas medicinais. Esse resultado vai de encontro ao estudo de Colet e colaboradores ${ }^{13}$, onde $81 \%$ dos entrevistados utilizavam plantas medicinais e ao estudo de Zeni e colaboradores ${ }^{14}$, onde $96 \%$ confirmaram a utilização. Ao observar que todos os entrevistados (100\%) informaram utilizar plantas medicinais, pode-se depreender que os produtos naturais possuem ampla aceitação, inferindo-se que possuem eficácia e legitimidade reconhecidas pela população estudada.

Outro fator que poderia ter contribuído para esse resultado é a facilidade de acesso, pois a maioria (64\%) dos entrevistados relatou possuir a planta cultivada na sua residência. Como foi observado em alguns estudos, as pessoas detentoras do conhecimento sobre recursos naturais se encontram na faixa etária de adulto a idoso ${ }^{15,14}$, podendo este ser um fator adicional que contribuiu com esse uso amplo, pois a população estudada se encontra na faixa etária mais avançada. Em relação a medicamentos fitoterápicos, apenas um (4\%) participante informou ter utilizado, pelo período de um ano.

Ao questionar se os entrevistados possuíam o hábito de repassar o seu conhecimento sobre plantas medicinais, $76 \%$ afirmaram que passam seu conhecimento adiante para amigos e familiares. Da mesma forma, costumam indicar as plantas com o motivo de querer ajudar/melhorar os sintomas de algum problema de saúde (95\%) e porque acreditam fazer bem (5\%). Tais resultados demonstram o aspecto tradicional na utilização das plantas medicinais, onde o conhecimento é passado de geração em geração e entre pessoas de uma comunidade. Outro dado encontrado no estudo reforça essa ideia, onde $92 \%$ dos entrevistados relataram ter recebido seu conhecimento a partir de parentes.

Ao questionar se com o uso da planta medicinal obtinha-se o efeito desejado, $68 \%$ afirmaram que sempre obtinham o efeito desejado, $28 \%$ obtinham "às vezes", pois em algumas situações necessitaram de medicamentos além das plantas, e em algumas situações utilizavam a planta medicinal sem estar com algum sintoma, portanto, não esperavam algum efeito. Ainda, 4\% afirmou nunca observar o efeito pois utilizava a planta apenas no chimarrão pelo sabor, sem esperar algum efeito benéfico. 0 resultado reforça a hipótese de que parte da aceitação vem da eficácia proporcionada pelo uso.

Entretanto, ao ser questionado sobre possíveis efeitos indesejados das plantas medicinais, 96\% dos participantes informaram não conhecer nenhum mal proveniente do uso de plantas. $4 \%$ informou que a marcela poderia causar alergias. Cruz e colaboradores ${ }^{16}$, encontrou resultado semelhante, onde $94 \%$ dos entrevistados, informaram não conhecer nenhum efeito indesejado das plantas medicinais. Ao questionar os entrevistados se acreditavam que as 
plantas medicinais poderiam possuir contraindicação, 64\% afirmaram que as plantas não possuem contraindicação e $32 \%$ afirmaram que sim, as plantas possuem contraindicação, sendo destes, 87,5\% afirmaram que o uso em excesso é contraindicado e 12,5\% afirmaram que é contraindicado o uso de plantas com medicamentos, pois pode interferir na ação destes. Tais resultados demonstram que a ideia de inocuidade dos produtos naturais é muito presente e constituem fator adicional para sua busca e utilização. Dado que estes conhecimentos foram recebidos por essas pessoas no seio da comunidade e são perpetuados, constituem fator que demanda atenção. Sabe-se que as plantas medicinais não são inócuas, podendo ocasionar reações adversas e interagir negativamente com fármacos ${ }^{17}$. Portanto, há uma oportunidade de ações de saúde que envolvam o esclarecimento sobre os riscos da utilização inadequada de produtos naturais, para garantir o uso seguro e racional destes produtos.

As plantas medicinais utilizadas pela população estudada se encontram na Tabela 1, junto com a indicação terapêutica citada pelos entrevistados e a indicação terapêutica que se encontra na literatura.

Tabela 1 - Plantas medicinais e suas indicações terapêuticas

\begin{tabular}{|c|c|c|c|c|}
\hline Nome Científico & $\begin{array}{l}\text { Nome } \\
\text { Popular }\end{array}$ & $\%$ & Indicação popular & Indicação Terapêutica Comprovada \\
\hline Achyrocline satureioides (Lam.) DC. & Marcela & 52 & Problemas de estômago & $\begin{array}{l}\text { Antidispéptico, antiespasmódico e } \\
\text { antiinflamatório. }\end{array}$ \\
\hline Matricaria chamomilla $L$. & Camomila & 44 & Calmante & $\begin{array}{c}\text { Antiespasmódico, ansiolítico e sedativo } \\
\text { leve, Anti-inflamatório } \\
\text { em afecções da cavidade oral. }\end{array}$ \\
\hline Plectranthus barbatus Andrews & Boldo & 24 & Problemas do fígado & $\begin{array}{l}\text { Colagogo, colerético e nas dispepsias } \\
\text { funcionais }\end{array}$ \\
\hline Mentha $L$. & Hortelã & 20 & Digestão e Antigripal & Antiespasmódico e anti flatulento \\
\hline "Baccharis trimera (Less.) & Carqueja & 12 & Digestão & Antidispéptico. \\
\hline Maytenus ilicifolia (Schrad.) Planch. & $\begin{array}{l}\text { Espinheira } \\
\text { santa }\end{array}$ & 12 & Dor, purifica sangue & $\begin{array}{c}\text { Antidispéptico, antiácido e protetor da } \\
\text { mucosa } \\
\text { gástrica }\end{array}$ \\
\hline Citrus L. & Laranjeira & 12 & Antigripal & Ansiolítico e sedativo leve \\
\hline Mikania glomerata Sprengel & Guaco & 12 & Tosse, Antigripal & Expectorante \\
\hline $\begin{array}{c}\text { Campomanesia xanthocarpa } 0 . \\
\text { Berg }\end{array}$ & Gabiroba & 12 & Colesterol, diabetes & - \\
\hline Pimpinella anisum $L$. & Erva doce & 12 & $\begin{array}{l}\text { Calmante, } \\
\text { Problemas no estômago, } \\
\text { substituir o café }\end{array}$ & Antidispéptico e antiespasmódico \\
\hline Cymbopogon citratus (DC.) Stapf & Cidreira & 12 & Calmante, gripe, estômago & $\begin{array}{c}\text { Antiespasmódico, ansiolítico e sedativo } \\
\text { leve }\end{array}$ \\
\hline Zingiber officinale Roscoe & Gengibre & 12 & Para uso no chimarrão & $\begin{array}{c}\text { Antiemético, antidispéptico, } \\
\text { expectorante e nos casos de cinetose. }\end{array}$ \\
\hline Melissa officinalis $L$. & Melissa & 8 & Calmante & $\begin{array}{c}\text { Antiespasmódico, ansiolítico e sedativo } \\
\text { leve }\end{array}$ \\
\hline Origanum majorana $L$. & Manjerona & 8 & Calmante, vaporização & - \\
\hline Aloe vera (L.) Burman $f$ & Babosa & 8 & Cicatrização, tratar infecções & Cicatrizante \\
\hline Rosmarinus officinalis $L$. & Alecrim & 8 & Hipertensão, antigripal & Antidispéptico e antiinflamatório \\
\hline Morus nigra L. & Amora & 4 & Menopausa & - \\
\hline Equisetum giganteum $L$. & Cavalinha & 4 & Diurético & $\begin{array}{c}\text { Edemas (inchaços) por retenção de } \\
\text { líquidos. } \\
\text { Coadjuvante no tratamento da hipertensão } \\
\text { leve }\end{array}$ \\
\hline
\end{tabular}




\begin{tabular}{|c|c|c|c|c|}
\hline Cynara scolymus L. & Alcachofra & 4 & Colesterol & $\begin{array}{l}\text { Auxiliar na prevenção da arterosclerose. } \\
\text { Coadjuvante no tratamento de dyslipidemia } \\
\text { mista leve a moderada e como auxiliar nos } \\
\text { sintomas da síndrome do intestine irritável }\end{array}$ \\
\hline Phyllanthus niruri L. & Quebra- pedra & 4 & Pedra nos rins & Litolítico nos casos de litíase urinária \\
\hline Casearia sylvestris Sw & $\begin{array}{c}\text { Erva- } \\
\text { dapontada }\end{array}$ & 4 & Antigripal & Antidispéptico \\
\hline Illicium verum Hook F & Anis estrelado & 4 & Chimarrão & Expectorante e anti flatulento \\
\hline Plantago major $L$. & Tanchagem & 4 & Antibiótico & $\begin{array}{c}\text { Anti-inflamatório e antisséptico da } \\
\text { cavidade } \\
\text { oral }\end{array}$ \\
\hline Malva sylvestris $L$. & Malva & 4 & Infecções & expectorante \\
\hline Carya illinoensis (Wang.) K & Noz-pecã & 4 & Cura & - \\
\hline Passiflora spp. & Maracujá & 4 & Calmante & Ansiolítico e sedativo leve \\
\hline Hibiscus & Hibisco & 4 & Intestino & - \\
\hline Cinnamomum verum J. Presl & Canela & 4 & Problemas no estômago & $\begin{array}{c}\text { Aperiente, antidispéptico, antiflatulento e } \\
\text { antiespasmódico }\end{array}$ \\
\hline $\begin{array}{c}\text { Stachytarpheta cayennensis (Rich.) } \\
\text { Vahl. }\end{array}$ & Gervão & 4 & Problemas no estômago & - \\
\hline Artemisia absinthium L. & Losna & 4 & Problemas no fígado & $\begin{array}{c}\text { Estimulante do apetite, epéptica, } \\
\text { antiespasmódica, anti dismenorréica }\end{array}$ \\
\hline Eugenia uniflora $L$. & Pitangueira & 4 & Diarreia & Antidiarreico em diarreia não infecciosa \\
\hline Cissus sicyoides $L$. & Insulina Natural & 4 & Controle da diabetes & \\
\hline
\end{tabular}

Fonte: Formulário de Fitoterápicos da Farmacopeia Brasileira ${ }^{22}$, 2018; Memento Fitoterápico ${ }^{23}, 2016$.

A espécie vegetal mais citada foi a marcela (52\%), seguido da camomila (44\%) e boldo $(24 \%)$, esses resultados se assemelham ao estudo realizado na cidade ljuí - RS, onde ocorreu 224 citações do uso da marcela, seguido de 171 citações para a camomila ${ }^{13}$, mas se difere de resultados encontrados na cidade Jequitinhonha - MG, onde obteve-se 277 citações para hortelã ${ }^{16}$. Resultados distintos também foram encontrados no município de Puxinanã (PB), onde a espécie vegetal mais citada foi a hortelã $(85,2 \%)$, seguido de erva de Santa Maria, pata de vaca e lípia, cada uma com $77 \%$. Pode-se perceber que cada região brasileira possui a sua característica, isto devido ao tipo de vegetação da região, aspectos culturais e a grande biodiversidade que acompanha o país ${ }^{18}$. A proximidade entre ljuí e Cruz Alta, associada à semelhança dos resultados, reforça a ideia da tradicionalidade de uso dentro de um determinado espaço geográfico.

Ao analisar as indicações citadas pelos entrevistados com as indicações encontradas na literatura, percebe-se que grande parte do conhecimento está correto, sendo as indicações mais prevalentes para distúrbios gastrointestinais, seguida de distúrbios do sistema nervoso. Esse conhecimento popular que condiz com as indicações terapêuticas comprovadas, reforça a hipótese de que parte do amplo uso que foi observado seja devido a eficácia obtida, o que contribui também para a transmissão e perpetuação desse conhecimento.

Entretanto, algumas plantas são utilizadas de forma incorreta pela população, como a erva-doce, mencionada como substituta do café. $\mathrm{O}$ café possui efeito estimulante devido sobretudo à presença de metilxantinas, enquanto a erva-doce tem o efeito antagônico. Este uso incorreto curiosamente também foi encontrado no estudo realizado na região de Colombo-PR ${ }^{19}$. 
As seguintes espécies vegetais foram indicadas pelos participantes como antigripais: hortelã, laranjeira, cidreira, alecrim e erva da ponta. Porém, na literatura pesquisada não foram encontradas indicações que corroborem aquela citada pelos entrevistados. Da mesma forma, não foram encontrados dados terapêuticos comprovados para as seguintes plantas citadas: gabiroba, manjerona, amora, noz, hibisco, gervão e insulina natural.

Em relação a forma de uso das plantas medicinais, a folha (92\%) foi a parte da planta mais citada para a utilização, seguido da flor (68\%) para marcela e camomila, a casca/caule (12) para canela, noz-pecã e cavalinha, as sementes (12\%) para a erva-doce e gel (4\%) para a babosa. O chá (80\%) foi o meio de consumo mais popular entre os entrevistados, seguido da utilização no chimarrão (20\%), para substituir o café (4\%) e na forma de uso externo (4\%). Em estudo realizado no município de Rio de Contas - BA foram encontrados resultados semelhantes, em que a grande parte dos entrevistados utilizava a folha $(37,86 \%)$ e o chá era a forma de preparo de maior prevalência $(66,25 \%)^{20}$. Estes resultados também condizem com a literatura, sendo a marcela e a camomila as plantas mais utilizadas, sua forma de uso também está correta em relação ao farmacógeno, utilizando as inflorescências. Considerando que folhas e flores são tecidos macios, prestam-se bem a processo de infusão, o qual também consiste em uma forma de preparo que é simples e de amplo uso tradicional.

Foi analisado o uso em associação de plantas medicinais com medicamentos industrializados e/ou manipulados. Entre os entrevistados, $76 \%$ afirmaram não utilizar plantas com medicamentos e $24 \%$ informaram que utilizam. As associações encontradas foram as seguintes: chá de amora com fluoxetina e Tandrilax®; chá de alcachofra com cálcio, levotiroxina sódica e omeprazol; chá de erva da pontada com antigripais; chá de folha de laranjeira com Benegripß e chá de marcela com medicamento para refluxo. Ao questionar sobre a interferência das plantas medicinais com os medicamentos utilizados em associação, todos (100\%) afirmaram acreditar que a associação não possui nenhum efeito negativo ou interferência. Neste trabalho, não foram encontradas indicações de interações entre as plantas medicinais apontadas e seus constituintes com os medicamentos citados. Entretanto, a atual ausência de dados a respeito não implica em inexistência de interações. Este fator é outro que se soma às possibilidades de esclarecimento para uso racional desses recursos.

Além das associações citadas, foram analisadas as possíveis interações das plantas medicinais com os medicamentos de uso atual mais citados pelos participantes. Os medicamentos mais utilizados se encontram na classe dos anti-hipertensivos (40\%). Das plantas medicinais utilizadas, carqueja, cidreira, melissa, alecrim, quebrapedra, tanchagem e losna, podem interferir na ação, aumentando o efeito hipotensor do medicamento, e o gengibre é contraindicado para pessoas com hipertensão arterial21.

A segunda classe de medicamento mais utilizado foi anti-inflamatório não esteroidal (28\%). Das plantas utilizadas, foi encontrado na literatura a interação do guaco com esta classe de medicamento, pois a utilização em conjunto pode 
interferir na coagulação sanguínea ${ }^{22}$. Foi encontrado contraindicação da melissa para pessoas com hipotireoidismo $22:$ no estudo, 20\% dos participantes informaram utilizar hormônio tireoidiano.

A utilização das plantas carqueja, gengibre e quebra-pedra concomitante com os medicamentos para diabetes deve ser evitada, pois essas plantas podem aumentar o efeito hipoglicêmico desses medicamentos ${ }^{22}$. No estudo, $12 \%$ informaram utilizar medicamento antidiabético.

Para as seguintes plantas, foi encontrado na literatura interação com medicamentos que interferem na coagulação sanguínea: maracujá, alcachofra, gengibre e camomila. No presente estudo, 12\% dos participantes informaram utilizar medicamentos para circulação, podendo então ser mais uma classe de medicamentos com potenciais interações com as plantas medicinais. A classe das estatinas foi citada por $8 \%$ dos participantes, a camomila possui interação com essa classe de fármacos 22,23 .

Os dados acima consolidam, portanto, a necessidade de ações de esclarecimento para o uso racional de plantas medicinais. Considerando que hipotensores e hormônios tireoideanos são de uso crônico, e as plantas medicinais são usados pela totalidade do grupo estudado, evidencia-se aí um grande risco de insucesso terapêutico e riscos à saúde acarretados pelo uso concomitante desses recursos.

Levando em consideração os temas já discutidos no presente trabalho, a prática da automedicação pelo uso de plantas medicinais associada ao uso de múltiplos fármacos, reforça-se a necessidade que o uso destes recursos terapêuticos seja orientado por profissionais de saúde. Em que pese o uso tradicional correto para muitas das plantas citadas, há um risco inerente ao seu uso, tanto individualmente quanto em associação, requerendo, portanto, atenção.

\section{CONSIDERAÇÕES FINAIS}

A partir deste estudo foi possível concluir que o uso de plantas medicinais possui lugar de destaque como alternativa terapêutica na população. Foi observado que a marcela e a camomila foram as plantas mais mencionadas pelos participantes, sendo os principais problemas tratados os distúrbios gastrointestinais e do sistema nervoso. Digno de nota é o fato que o uso popular da maioria das plantas citadas coincide com as indicações terapêuticas comprovadas. Entretanto, pode-se observar que há pouco conhecimento pela população sobre efeitos indesejados dos produtos naturais, assim como, utilizam as plantas medicinais em associação com medicamentos alopáticos sem a preocupação de possíveis interações e efeitos adversos. 


\section{REFERÊNCIAS}

1. World Health Organization. Traditional Medicine Strategy. Geneva: WHO, 1998.

2. Brasil. Ministério da Saúde (MS). Resolução da Diretoria Colegiada-RDC n² 26, de 13 de maio de 2014. Dispõe sobre o registro de medicamentos fitoterápicos e o registro ea notificação de produtos tradicionais fitoterápicos, junto à Agência Nacional de Vigilância Sanitária. Brasília, 2014.

3. Moraes EF, Mezzomo TR, Oliveira VB. Conhecimento e uso de plantas medicinais por usuários de unidades básicas de saúde na região de Colombo, PR. Revista Brasileira de Ciências da Saúde. 2018;22:57-64.

4. Rodrigues LS, Da Silva ARA, Macêdo AAM. Noni (Morinda citrifolia Linn.): Determinação Fitoquímica e Potencial Antioxidante pelo Método DPPH. Conexões Ciência e Tecnologia. 2017;11: 47-54.

5. Martins MC, Garlet TMB. Desenvolvendo e divulgando o conhecimento sobre plantas medicinais. Revista Eletrônica em Gestão, Educação e Tecnologia Ambiental. 2016;20:438-448.

6. Fernandes WS, Cembranelli JC. Automedicação e o uso irracional de medicamentos: o papel do profissional farmacêutico no combate a essas práticas. Revista Univap. 2015; 21:5-12.

7. Ângelo T, Ribeiro CC. Utilização de plantas medicinais e medicamentos fitoterápicos por idosos. Ciência \& Desenvolvimento-Revista Eletrônica da FAINOR. 2014; 7:18-31.

8. Júnior JMA, Salvi JO. Fatores associados à automedicação em uma farmácia comunitária de ouro preto do oeste, Rondônia. Acta Biomedica Brasiliensia. 2018;9:107-116.

9. Piriz MA, Mesquita MK, Cavada CT, Palma JS, Ceolin T, Heck RM. Uso de plantas medicinais: impactos e perspectivas no cuidado de enfermagem em uma comunidade rural. Rev Eletr de Enferm. 2013; 15: 992-999.

10. Souza LAF et al. Prevalência e caracterização da prática de automedicação para alívio da dor entre estudantes universitários de enfermagem. Rev. Latinoam. Enfermagem. 2011;19:245-251. 
11. Ferreira RL, Júnior ATT. Estudo sobre a automedicação, o uso irracional de medicamentos e o papel do farmacêutico na sua prevenção. Revista Científica da Faculdade de Educação e Meio Ambiente. 2018;9:570-576.

12. Paixão JA et al. Levantamento bibliográfico de plantas medicinais comercializadas em feiras da Bahia e suas interações medicamentosas. Revista Eletrônica de Farmácia. 2016;13:71-81.

13. Colet C, Cavalheiro CAN, Dal Molin GT, Cavinato AW, Schiavo M, Schwambach KH, et al. Uso de plantas medicinais por usuários do serviço público de saúde do município de ljuí/RS. Revista Brasileira de Medicina de Família e Comunidade. 2015;10:1-13.

14. Zeni ALB, et al. Utilização de plantas medicinais como remédio caseiro na Atenção Primária em Blumenau, Santa Catarina, Brasil. Ciência \& Saúde Coletiva. 2017;22:2703-2712.

15. Stefanello S, Kozera C, Ruppelt BM, Fumagalli D, Camargo MP, Sponciado D. Levantamento do uso de plantas medicinais na Universidade Federal do Paraná, Palotina-PR, Brasil. Extensão em Foco. 2018;1:15-27.

16. Cruz MJB, et al. Uso de plantas medicinais por famílias do Vale do Jequitinhonha, Minas Gerais, Brasil. Infarma-Ciências Farmacêuticas. 2015;27:38-48.

17. WORLD HEALTH ORGANIZATION (WHO). 2002. The World Medicines Situation Report. Disponível em: <http://www.who.int/medicines/areas/policy/world_medicines_situation/wms_intro/en/i ndex.html. > Acessado em: outubro de 2018.

18. Lima Filho JA, Marinho MGV. Levantamento da diversidade e uso das plantas medicinais utilizadas por moradores do município de Puxinanã, PB, Brasil. Gaia Scientia Ed. Esp. Populações Tradicionais. 2014; 229-249.

19. Oliveira VB, Mezzomo TR, Moraes EF. Conhecimento e uso de plantas medicinais por usuários de unidades básicas de saúde na região de Colombo, PR. Revista Brasileira de Ciências da Saúde. 2018;22:57-64.

20. Ramos ES, Damascena R S. Avaliação do Uso de Plantas Medicinais na Academia da Saúde do Município de Rio de Contas/BA. Id on Line Revista de Psicologia. 2018;12:75-84. 
21. Teixeira K, dos Santos P, Citadini-Zanette V, et al. Medicinal Plants that can Cause Changes in Blood Pressure and Interactions with Antihypertensive Agents. Am J Ethnomed. 2017, 4:1.

22. Brasil. Agência Nacional de Vigilância Sanitária. Formulário de Fitoterápicos da Farmacopéia Brasileira. Brasília, 2018.

23. Brasil. Agência Nacional de Vigilância Sanitária. Memento Fitoterápico. Brasília, 2016. 